\title{
Widely Tunable Silicon Mid-Infrared Optical Parametric Oscillator
}

\author{
Bart Kuyken ${ }^{1,2 *}$, Xiaoping Liu ${ }^{3}$, Richard M. Osgood, Jr. ${ }^{3}$, Roel Baets ${ }^{1,2}$ \\ Günther Roelkens ${ }^{1,2}$, and William M. J. Green ${ }^{4, \dagger}$ \\ ${ }^{1}$ Photonics Research Group, Department of Information Technology, Ghent University - imec, Ghent, Belgium \\ ${ }^{2}$ Center for Nano- and Biophotonics (NB-Photonics), Ghent University, Ghent, Belgium \\ ${ }^{3}$ Department of Electrical Engineering, Columbia University, 1300 S. W. Mudd, 500 W. 120th St., New York, NY 10027, USA \\ ${ }^{4}$ IBM Thomas J. Watson Research Center, Yorktown Heights, NY 10598, USA \\ *Bart.Kuyken@intec.ugent.be, ${ }^{\dagger}$ wgreen@us.ibm.com
}

\begin{abstract}
A synchronously-pumped mid-infrared optical parametric oscillator is demonstrated using unprecedented large parametric gain in silicon photonic wires. The output wavelength is tunable over a $75-\mathrm{nm}$ bandwidth, with a maximum output pulse energy of $1.62 \mathrm{pJ}$.

OCIS codes: (190.4380) Four wave mixing; (130.4310) Nonlinear integrated optics
\end{abstract}

\section{Introduction}

The silicon nanophotonic integrated circuit platform continues to undergo extensive technological development for numerous applications within the telecommunication wavelength range. Moreover, many applications operating in the short-wave infrared to mid-infrared (mid-IR) also stand to benefit from this integration technology, with integrated spectroscopy and free-space communication systems being some examples. High index contrast siliconon-insulator waveguide circuits show very high third-order optical nonlinearity, due to the high confinement of the optical mode and the large material $\chi^{(3)}$ in silicon. For wavelengths within the $2 \mu \mathrm{m}$ mid-IR range, silicon photonic integrated circuits become interesting for nonlinear applications, given the absence of the parasitic two-photon absorption process. This has led to the demonstration of very large four-wave mixing-based nonlinear parametric gain in centimeter-scale silicon photonic wires $[1,2]$. Here for the first time, we utilize this large gain to realize a broadband tunable silicon mid-IR optical parametric oscillator.

\section{Silicon photonic wire design and single-pass parametric gain}

The silicon nanophotonic wires used in the experiment are fabricated on $200 \mathrm{~mm}$ SOI wafers in a CMOS pilot line, consisting of a $220 \mathrm{~nm}$ silicon waveguide layer on a $2 \mu \mathrm{m}$ buried oxide layer. The wires are $900 \mathrm{~nm}$ wide with no top cladding, as shown in the inset of Figure 1(a). The high confinement realized in these silicon nanophotonic wires not only results in a high effective nonlinearity parameter $\left(\gamma \sim 150 \mathrm{~W}^{-1} \mathrm{~m}^{-1}\right)$, but also gives rise to anomalous waveguide dispersion $\left(\beta_{2}<0\right)$ in the mid-IR, as required for phase-matching and large parametric gain. Figure 1(a) plots the dispersion for the fundamental TE polarized mode, which is anomalous between the two zero dispersion wavelengths of $1800 \mathrm{~nm}$ and $2410 \mathrm{~nm}$. Cut-back measurements show that the waveguide propagation loss is approximately $1 \mathrm{~dB} / \mathrm{cm}$ across the mid-IR region.
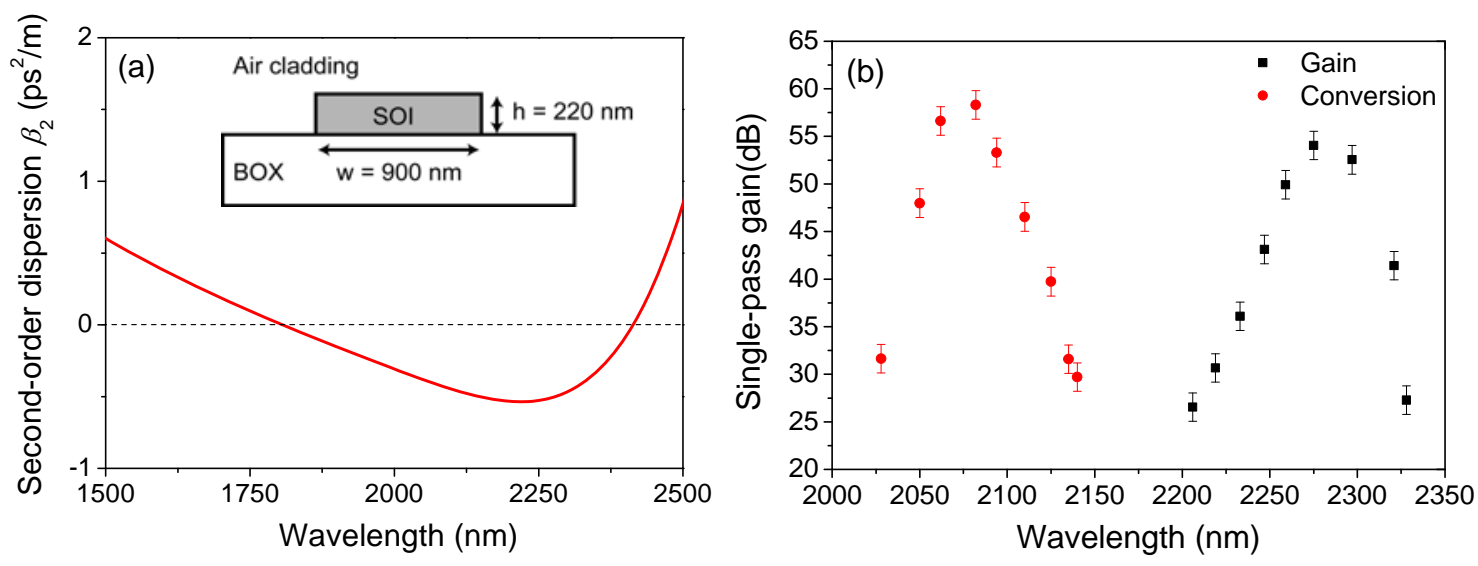

Figure 1: (a) Dispersion of the silicon photonic wire, simulated using a commercial finite element solver (RSoft FemSIM). (b) Single-pass gain and conversion in the $2 \mathrm{~cm}$ photonic wire for pump pulses at $2175 \mathrm{~nm}$ having an energy of $48 \mathrm{pJ}$ (24W peak power). 
The single-pass gain of a $2 \mathrm{~cm}$ long silicon waveguide is first characterized [1], using a picosecond pump pulse train $(\mathrm{FWHM} \sim 2 \mathrm{ps}$, repetition rate $=76 \mathrm{MHz}$ ) at a center wavelength of $2175 \mathrm{~nm}$, having a waveguide-coupled input pulse energy of $48 \mathrm{pJ}$ (peak power $24 \mathrm{~W}$ ). Coupling into/out of the SOI photonic wire is accomplished using lensed fibers, with a coupling loss of $7 \mathrm{~dB}$ at each chip facet. A polarization controller is used to excite the fundamental quasi-TE-polarized mode of the photonic wire. Single-pass parametric gain and wavelength conversion efficiency as large as $54 \mathrm{~dB}$ and $58 \mathrm{~dB}$ respectively, are shown in Figure 1(b). Net on-chip gain is obtained over a mid-IR bandwidth greater than $300 \mathrm{~nm}$.

\section{Synchronously pumped tunable optical parametric oscillator}

This broadband on-chip parametric gain available from the silicon photonic wires can be combined with optical feedback in order to achieve optical parametric oscillation over a broad wavelength range. The synchronouslypumped fiber optic loop configuration employed to construct the mid-IR OPO is illustrated in Figure 2. The mid-IR pumped photonic wire serves as the core gain element within the fiber loop. The cavity of the OPO is formed by a loop of standard single mode fiber, along with a variable free-space delay element, which facilitates temporal synchronization of the re-circulating amplified pulses with successive pump pulses. Due to the absence of specialpurpose wavelength multiplexers/demultiplexers within the mid-IR wavelength range of operation, a 90/10 coupler is used to combine the cavity pulses with successive pump pulses after each round-trip. Polarization controllers are used to align both the pump and cavity pulses to the quasi-TE-mode at the input of the photonic wire. The output of the silicon-fiber OPO is monitored by a mid-infrared optical spectrum analyzer (OSA).

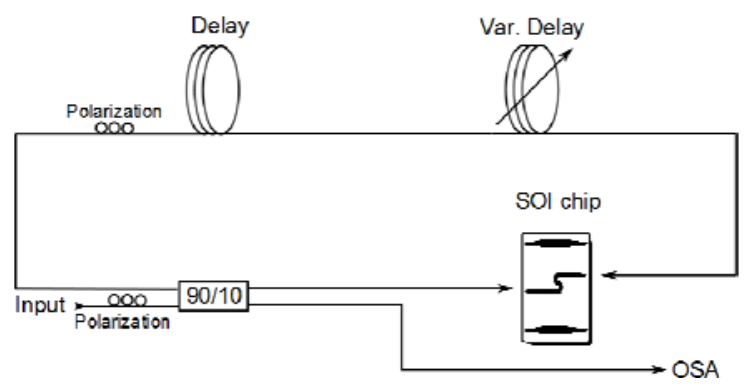

Figure 2: Configuration of the synchronously-pumped silicon-fiber OPO.

The highly wavelength-dependent cavity round-trip loss shown in Figure 3 originates from the insertion loss of the silicon chip, as well as large losses within the variable delay line and 90/10 coupler, particularly at longer wavelengths. Nevertheless, comparison with the gain spectrum in Figure 1(b) illustrates that the gain substantially exceeds the round-trip losses within a wide band around $2075 \mathrm{~nm}$, and also within a narrower region around 2280 $\mathrm{nm}$. Figure 4(a) shows the output spectrum for increasing pump pulse energies, when the fiber cavity is configured for an oscillation wavelength of $2075 \mathrm{~nm}$. The $3 \mathrm{~dB}$ spectral bandwidth of the oscillator's output pulses (at maximum output power) is $7.5 \mathrm{~nm}$, equal to the $3 \mathrm{~dB}$ spectral bandwidth of the input pump. In Figure 4(b), the threshold pump energy for parametric oscillation is determined to be approximately $17 \mathrm{pJ}$, while the slope efficiency is $5.4 \%$. It is believed that the saturation in output energy is the result of nonlinear losses arising from two-photon absorption and free carrier absorption at these high on-chip powers.

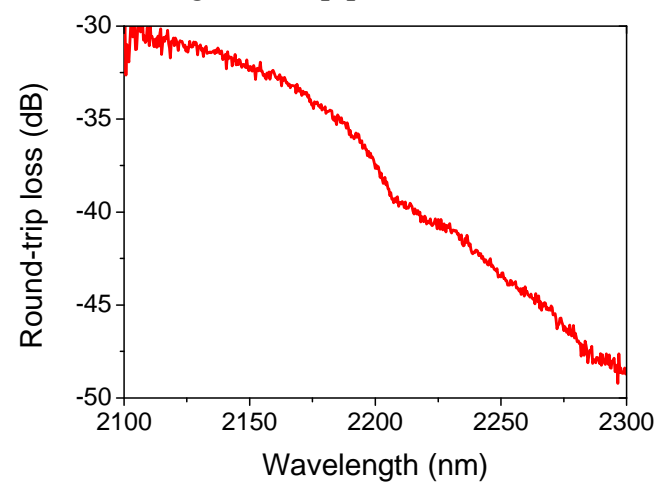

Figure 3: Wavelength-dependent cavity round-trip loss. 
The high dispersion of the single mode fiber feedback loop at wavelengths beyond $2000 \mathrm{~nm}$ allows for wavelength selective feedback. By adjusting the round-trip time using the free-space variable delay line, a particular temporal "slice" of the circulating amplified pulse is selectively synchronized with the pump pulse train. The dispersion in the fiber loop likewise ensures that only one particular wavelength within the silicon parametric gain spectrum can be synchronized with the pump pulses, and thus enables broadband tunability of the oscillator wavelength via delay tuning. The energy of the output pulses as a function of the output wavelength for pump pulses with an energy of $48 \mathrm{pJ}$ at $2175 \mathrm{~nm}$ is shown in Figure 5. It can be seen that the OPO is tunable over a $75 \mathrm{~nm}$-wide band centered around the gain peak at $2075 \mathrm{~nm}$. Despite higher cavity losses, the silicon-fiber OPO can additionally be tuned to operate at longer wavelengths near $2270 \mathrm{~nm}$, at which pulses of up to $0.12 \mathrm{pJ}$ can be generated.
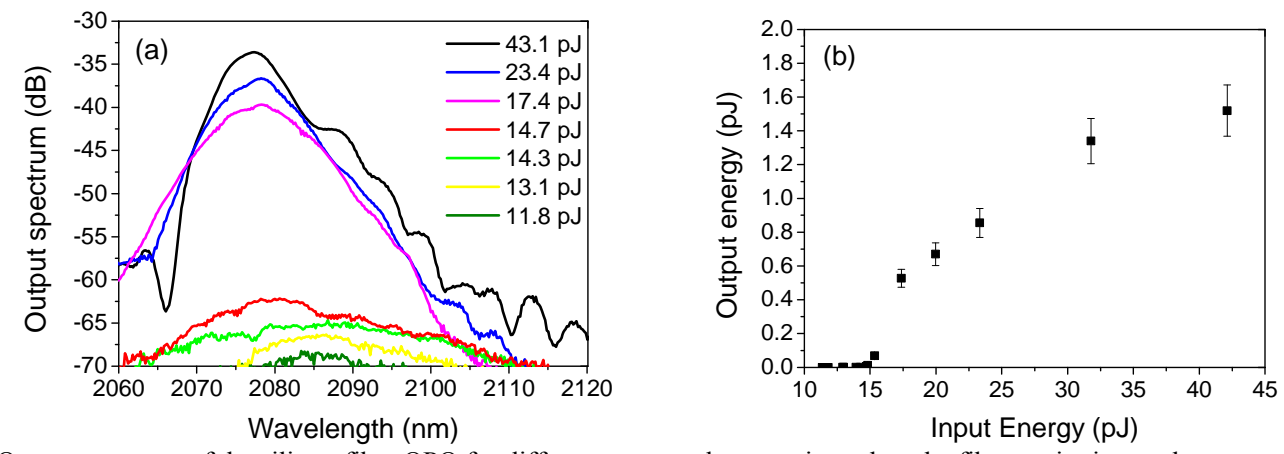

Figure 4: (a) Output spectrum of the silicon-fiber OPO for different pump pulse energies, when the fiber cavity is tuned to an output wavelength of $2075 \mathrm{~nm}$. (b) On-chip output pulse energy versus the coupled input pump pulse energy.

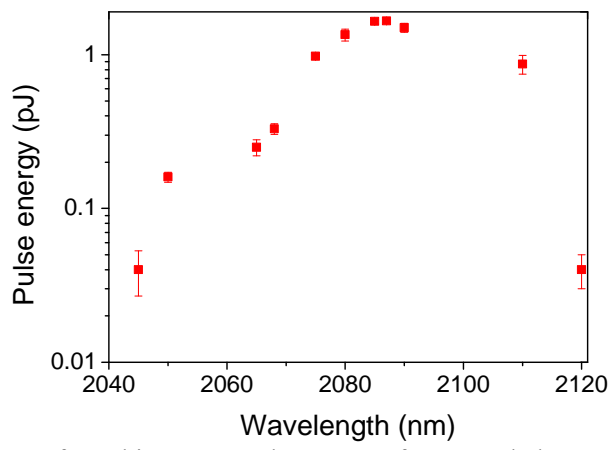

Figure 5: Tuning spectrum of on-chip output pulse energy, for a coupled pump energy of $48 \mathrm{pJ}$ at $2175 \mathrm{~nm}$.

\section{Conclusions}

In summary, a widely tunable mid-IR optical parametric oscillator was demonstrated based on a silicon waveguide as the nonlinear gain element. The wavelength can be tuned over a $75 \mathrm{~nm}$ band around $2075 \mathrm{~nm}$, but can also operate at longer wavelengths. Optimization of the fiber-chip coupling losses, fiber losses, and the mid-IR multiplexers can give rise to improvements in the oscillation threshold energy and slope efficiency. Since these factors are not fundamental, a wavelength tuning range of over $500 \mathrm{~nm} \mathrm{[2]} \mathrm{could} \mathrm{in} \mathrm{principal} \mathrm{be} \mathrm{obtained.} \mathrm{Another}$ appealing solution is the ultimate integration of optical feedback on the silicon chip, to make an on-chip OPO [3-5].

The authors would like to acknowledge Yurii A. Vlasov of IBM Research for many constructive technical discussions during the course of this work.

[1] X. P. Liu, R. M. Osgood, Y. A. Vlasov, and W. M. J. Green, "Mid-infrared optical parametric amplifier using silicon nanophotonic waveguides," Nat. Photon. 4, 557-560 (2010).

[2] X. P. Liu, B. Kuyken, G. Roelkens, R. Baets, Y. Vlasov, R. M. Osgood Jr., W. Green, "Mid-infrared broadband modulation instability and 50 dB Raman assisted parametric gain in silicon photonic wires," CLEO 2011, paper CTuS2.

[3] Levy, J. S. et al. "CMOS-compatible multiple-wavelength oscillator for on-chip optical interconnects," Nat. Photon. 4, 37-40 (2010).

[4] L. Razzari, D. Duchesne, M. Ferrera, R. Morandotti, S. Chu, B. E. Little \& D. J. Moss, "CMOS-compatible integrated optical hyperparametric oscillator," Nat. Photon. 4, 41 - 45 (2010).

[5] P. Del'Haye, A. Schliesser, O. Arcizet, T. Wilken, R. Holzwarth \& T. J. Kippenberg, "Optical frequency comb generation from a monolithic microresonator," Nature 450, 1214-1427 (2007). 